\title{
"Rehearsing" according to artificially generated rehearsal patterns: An analysis of active rehearsal
}

\author{
PETER A. ORNSTEIN \\ University of North Carolina, Chapel Hill, North Carolina \\ and \\ MARY J. NAUS \\ University of Houston, Houston, Texas
}

\begin{abstract}
In order to examine directly the relationship between rehearsal strategies and recall performance, a "read aloud" procedure was developed such that subjects could "rehearse" by reading aloud specially prepared rehearsal protocols. With this procedure, it was possible to vary the degree of rehearsal activity represented in rehearsal, as measured by the number of different items rehearsed together, while holding constant the actual frequency of item rehearsal. The data indicated that, even with rehearsal frequency equated, increases in rehearsal activity were associated with increases in recall. There was, however, a boundary condition to this rehearsalactivity/recall relationship, such that rehearsing six or seven items together did not result in performance superior to that obtained when three or four items were rehearsed together. In addition, the equivalence of the recall of control subjects who rehearsed themselves and of the recall of those who read the rehearsal protocols of others suggests that item retrieval is probably not an essential component of active rehearsal.
\end{abstract}

Research on the memory performance of adults and children has stressed the importance of rehearsal for effective list learning. Initial work suggested that rehearsal frequency was an important determinant of recall (e.g., Rundus, 1971; Rundus \& Atkinson, 1970), such that items that differ in the number of times they are rehearsed also differ in the probability of recall. In contrast, more recent studies (e.g., Craik \& Watkins, 1973; Ornstein, Naus, \& Liberty, 1975) have suggested that the type of rehearsal is more important than the amount of rehearsal in mediating recall. Rehearsal that is active and elaborative, as opposed to passive and simply repetitive, is thought to facilitate recall. Evidence for the importance of rehearsal activity comes from both the adult and developmental literatures.

In the adult literature, evidence for the activity hypothesis comes from studies that have indicated essentially that rehearsal frequency is not a powerful variable (e.g., Craik, 1973; Craik \& Watkins, 1973; Jacoby \& Bartz, 1972; Roenker, 1974; Woodward, Bjork, \& Jongeward, 1973). For example, in the context of his critique of multistore models of memory,

The research reported in this paper was supported by Grant HD 08459 from the U.S. Public Health Service. Requests for reprints should be sent to Peter A. Ornstein, Department of Psychology, Davie Hall 013A, University of North Carolina, Chapel Hill, North Carolina 27514.
Craik (1973; Craik \& Watkins, 1973) demonstrated that increases in the frequency of item rehearsal are not always associated with corresponding changes in recall probability. On the basis of this finding, the existence of two types of rehearsal was postulated, one of which was thought only to maintain information in primary memory and the other to lead to more permanent encoding as well. This second type of rehearsal was termed "elaborative" because it was felt to involve actively elaborating upon the to-be-remembered materials. Whereas Craik's data suggested that rehearsal frequency may not in itself be a major determinant of recall, there is no direct support for the view that changes in the degree of rehearsal activity (i.e., more or less elaboration) lead to changes in recall performance.

In contrast to the work with adult subjects, developmental investigations of rehearsal permit more direct statements about the importance of active types of rehearsal. Whereas rehearsal frequency and activity typically covary in adults (i.e., items that are rehearsed frequently tend also to be rehearsed actively with other items), developmental studies provide a way of separating these variables. For example, Ornstein et al. (1975) demonstrated that the items from the middle and terminal serial positions were rehearsed more by third-graders than by older children, and yet these items were recalled less well. Furthermore, the third-graders rehearsed the beginning items more than the middle items, but there 
were no differences in the recall of these two classes of items. However, it was found that the third-graders' rehearsal was less active than that of the older subjects, in that the third-graders tended to repeat the currently presented word either alone or in minimal juxtaposition with other items; in contrast, the rehearsal of older children was more active, in that it involved rehearsing each item in the context of other list members. In addition, training studies confirmed the linkage between rehearsal activity and recall performance (Naus, Ornstein, \& Aivano, 1977; Ornstein, Naus, \& Stone, 1977). For example, by manipulating rehearsal activity through instructions, and by providing variations in processing time per item, Naus et al. (1977) were able to create situations in which rehearsal frequency and rehearsal activity made differential predictions. Regardless of age, recall probability varied with rehearsal activity, not with rehearsal frequency.

Although the developmental literature indicates that rehearsal activity is critically related to recall, there have been no corresponding studies with adult subjects. A rehearsal training study, pitting rehearsal frequency against rehearsal activity, could easily be executed with adult subjects. However, a more definitive account of the rehearsal/recall relationship might stem from a situation in which the rehearsal frequency of individual items was specifically controlled. The present experiments were thus designed to demonstrate the effects of changes in rehearsal activity under conditions in which rehearsal frequency was held constant. This was accomplished by designing a task in which subjects read aloud rehearsal protocols that had been generated either artificially or by other subjects, rather than rehearsing spontaneously. Two experiments using this "read aloud" procedure are reported.

\section{EXPERIMENT 1}

Prior to examining variations in rehearsal activity per se, it was necessary to demonstrate that the recall of subjects "rehearsing" by reading aloud various rehearsal protocols would be comparable to that of subjects in typical free-recall situations. Accordingly, this experiment involved a comparison of subjects rehearsing on their own with those who read aloud from the rehearsal protocols of others.

\section{Method}

Design and Materials. Two experimental conditions, in which subjects learned a list of 20 unrelated words over a series of four alternating study/recall trials, were established. The subjects in a protocol-generating condition were visually presented with a new word on a $3 \times 5$ in. card approximately every $5 \mathrm{sec}$. These subjects were free to rehearse the words in any way desired, with the constraint that rehearsal be timed according to the beat of a metronome. One utterance was required every $.75 \mathrm{sec}$, and thus seven rehearsals were made as each word was presented. The rehearsal protocols of four of these subjects were selected to provide the basis for the "rehearsal" for subjects in a read- aloud condition. The subjects in this group were shown complete rehearsal sets (i.e., that rehearsal associated with the presentation of particular items, one at a time), printed on cards. Each of these rehearsal sets was displayed for approximately $5 \mathrm{sec}$, and the subjects were required to read the words at a .75-sec rate.

To partially control for list-specific effects, two lists of 20 unrelated high-frequency items (Thorndike \& Lorge, 1944) were selected. One-half of the subjects in the protocol-generating group received each of these lists. Four different random orders of presentation were generated for each list, and the subjects received these orders in unique sequences. The rehearsal of all of the protocol-generating subjects was recorded, and the rehearsal protocols of two representative subjects who had learned each of the lists were selected for the read-aloud subjects. Each of the four protocols was given to four subjects in the readaloud group.

Procedure. Each subject was tested individually by a female experimenter. The subjects in the protocol-generating group were told that they were to learn a list of common nouns by practicing the presented words aloud to the beat of a metronome. These subjects were free to rehearse any list item during the $5 \mathrm{sec}$ that each to-be-remembered word was displayed. The subjects in the read-aloud group also were told that they were to learn a list of words, but they were instructed that the words would be presented on cards in various groupings, and that they should read the words on each card aloud to the beat of the metronome. To accustom them to pacing their rehearsal or reading, all subjects were given practice lists appropriate to their conditions. After each list had been presented, $40 \mathrm{sec}$ was allowed for free recall.

Subjects. A total of 30 undergraduate students at the University of North Carolina, half of them male and half female, were paid for their participation in the study. There were 14 subjects in the protocol-generating group and 16 in the read-aloud group.

\section{Results and Discussion}

The subjects experienced no difficulty in rehearsing and reading rehearsal protocols aloud to the beat of the metronome. Furthermore, the recall performance of the protocol-generating and read-aloud groups was comparable. The subjects in the protocol-generating group recalled an average of 11.30 words, whereas those in the read-aloud condition recalled 12.45 words. An analysis of variance performed on these data, with rehearsal as a between-subjects factor and trials as a within-subjects factor, yielded only an effect due to trials $[F(3,84)=$ $71.48, \mathrm{p}<.001]$, reflecting improvement across the series of four trials. In addition, both groups also had age-appropriate serial position functions, with clear primary and recency effects. The equality of recall performance in the protocol-generating and read-aloud groups suggests that the reading-aloud technique can be used in subsequent experiments on rehearsal to explore the effects of rehearsal activity.

\section{EXPERIMENT 2}

To analyze the role of rehearsal activity in memory, all subjects in Experiment 2 read aloud specifically constructed rehearsal sets. These sets were generated such that the overall frequency of item rehearsal was equated across conditions that differed systematically 
in the extent to which different items were rehearsed together. It was thought that recall would increase as a function of the number of unique items present in each rehearsal set, but that there might be boundary conditions to this rehearsal/recall relationship. On the basis of Naus et al.'s (1977) finding that the recall of sixthgraders was not facilitated by increases in rehearsal set size beyond approximately 3.5 items, it was felt that increases in adult recall would not be observed under conditions in which more than four items were rehearsed together. Furthermore, to explore the Naus et al. (1977) suggestion that additional characteristics of rehearsal other than the number of unique items per rehearsal set are important for recall, the internal positioning of items was manipulated in some conditions. If the internal positioning of items in each rehearsal set were critical, then the groups in which there was maximal intermixing among the items during rehearsal should show enhanced recall vis à vis the groups in which there was minimal intermixing.

\section{Method}

Design and Materials. Six groups of 12 subjects each were established. The subjects in these conditions were provided different types of artificial rehearsal protocols generated from the four protocols selected in Experiment 1 for the read-aloud group. The subjects were asked to learn a list of 20 unrelated words by reading aloud, to the beat of a metronome, the protocols presented them. The protocols were constructed such that each contained seven utterances per rehearsal set, and they were equated in terms of overall frequency of item rehearsal. For example, if the first word had been rehearsed 20 times across all rehearsal sets on a given trial by a subject who generated one of the standard protocols, then in each of the six artificial protocols created from this protocol, it appeared 20 times. What did vary, however, were the extent to which different items were to be rehearsed together in each rehearsal set and, for some of the groups, the degree to which items were intermixed.

The six groups that were generated were as follows: (1) baseline, one or two different items represented in each rehearsal set; (2) three items included in each set, but represented in minimal combination (e.g., AABBCC); (3) three items represented in each set, but included in maximal combination (e.g., ABCBAC); (4) four items, minimally intermixed; (5) four items, maximally intermixed; and (6) six or seven items per set. The subjects in the six groups were presented with cards on which the entire rehearsal sets were printed. As had been the case in Experiment 1 , each rehearsal set was displayed for approximately $5 \mathrm{sec}$, and the subjects were required to read the words at a $.75-\mathrm{sec}$ rate, according to the beat of a metronome. To partially avoid list-specific effects, there were four versions of the lists presented in the six groups, one version being generated from each of the four protocols selected for the read-aloud subjects in Experiment 1. Three of the subjects assigned to each condition received each of the alternate lists.
Procedure. Each subject was tested individually by a male experimenter and was given instructions similar to those given to the read-aloud group in Experiment 1 . The conditions of stimulus presentation and recall were also similar.

Subjects. A total of 72 undergraduate students at the University of North Carolina, half of them male and half female, were paid for their participation in the experiment.

\section{Results and Discussion}

As had been the case in Experiment 1, the subjects were easily able to read the prepared rehearsal protocols according to the beat of the metronome. In addition, recall performance was basically in accord with expectations. As can be seen in Table 1, with reference to the baseline condition, mean recall performance increased with increases in rehearsal set size, up to sets of 4 items. There was also some indication that maximal intermixing of the items, when three and four items per rehearsal set were rehearsed, was superior to minimal intermixing. With the exception of the latter point, these observations were confined by the statistical analyses. In an analysis of variance carried out on these data, with rehearsal condition as a between-subjects factor and trials as a within-subjects factor, there were effects due to rehearsal condition $[\mathrm{F}(5,66)=2.61, \mathrm{p}<.05]$ and trials $[F(3,198)=159.71, p<.01]$, but there was no interaction between these variables. Furthermore, subsequent tests indicated that the mean recall for the three- and four-item groups (13.57 words) was superior to that for the baseline group. In addition, there was support for the prediction that there might be a boundary condition to the rehearsal-activity/recall relationship. The recall of the subjects who rehearsed six and seven items was not different from that of those who rehearsed three and four items. Finally, although the data were suggestive of elevated recall under conditions of maximal intermixing, the overall differences between these conditions did not reach significance.

\section{GENERAL DISCUSSION}

The results of these experiments were basically consistent with expectations. The findings of Experiment 1 suggested that the read-aloud procedure might be a valuable tool in the investigation of those properties of rehearsal that mediate performance. The findings of Experiment 2, in which the frequency of rehearsal was held constant, suggested clear effects of rehearsal activity. Taken together with the Naus et al. (1977) and Ornstein et al. (1977) experiments, substantial support was obtained for the view that rehearsal activity, measured in terms of the number of unique items rehearsed together, directly mediates recall. In addition, any statement that rehearsal activity facilitates recall

Table 1

Mean Recall as a Function of Condition

Rehearsal Set Composition

\begin{tabular}{|c|c|c|c|c|c|}
\hline & $\begin{array}{c}\text { Minimal } \\
\text { Intermixing }\end{array}$ & $\begin{array}{c}\text { Maximal } \\
\text { Intermixing }\end{array}$ & $\underset{\text { Intermixing }}{\text { Minimal }}$ & $\begin{array}{c}\text { Maximal } \\
\text { Intermixing }\end{array}$ & \\
\hline 1-2 Items & 3 Items & 3 Items & 4 Items & 4 Items & 6-7 Items \\
\hline 12.06 & 12.75 & 13.42 & 13.98 & 14.13 & 13.48 \\
\hline
\end{tabular}


needs to be qualified both by the specification of boundary conditions indicating limits on the number of items that can be effectively rehearsed together and by the possible importance of within-rehearsal-set variation in item position.

Finally, the use of the rehearsal-aloud paradigm also permits a comment on the degree to which the effects of active elaborative rehearsal may be mediated by practice in the retrieval of items to be rehearsed. One hypothesis concerning the benefits of active rehearsal might stem from the fact that active rehearsal demands the retrieval of previously presented items for inclusion in subsequent rehearsal sets (see Gates, 1917, and Slamecka \& Graf, 1978). To the extent to which retrieval per se may be one of the factors underlying the efficacy of active rehearsal, however, it would have been expected that the protocol-generating subjects in Experiment 1 would have recalled more than the read-aloud subjects. The subjects in the protocol-generating group, by definition, had to retrieve previously displayed items in order to rehearse, whereas the retrieval operation was minimized by the presentation of complete rehearsal sets for the read-aloud subjects. The equivalence of the recall of these two groups suggests that the retrieval of items to be rehearsed may not be an essential component of active rehearsal. It would seem, then, that other aspects of active rehearsal-perhaps the associations or organization resulting from the intermixing of items (see Ornstein \& Naus, 1978)-are responsible for its effects on recall performance.

\section{REFERENCES}

Craik, F. I. M. A "levels of analysis" view of memory. In P. Pliner, L. Krames, \& T. M. Alloway (Eds.), Communication and affect: Language and thought. New York: Academic Press, 1973.

Craik, F. I. M., \& Watkins, M. J. The role of rehearsal in shortterm memory. Journal of Verbal Learning and Verbal Behavior, 1973, 12, 599-607.
Gates, A. I. Recitation as a factor in memorizing. Archives of Psychology, 1917, 6, No. 40.

JACOBY, L. L., \& BARTz, W. A. Rehearsal and transfer to LTM. Journal of Verbal Learning and Verbal Behavior, 1972, 11, 561-565.

Naus, M. J., Ornstein, P. A., \& Aivano, S. Developmental changes in memory: The effects of processing time and rehearsal instructions. Journal of Experimental Child Psychology, 1977, 23, 237-251.

Ornste in, P. A., \& Naus, M. J. Rehearsal processes in children's memory. In P. A. Ornstein (Ed.), Memory development in children. Hillsdale, N.J: Erlbaum, 1978.

Ornstein, P. A., Naus, M. J., \& Liberty, C. Rehearsal and organizational processes in children's memory. Child Development, 1975, 46, 818-830.

Ornste in, P. A., Naus, M. J., \& Stone, B. P. Rehearsal training and developmental differences in memory. Developmental Psychology, 1977, 13, 15-24.

ROENKE R, D. L. Role of rehearsal in long-term retention. Journal of Experimental Psychology, 1974, 103, 368-371.

RUNDUs, D. Analysis of rehearsal processes in free recall. Journal of Experimental Psychology, 1971, 89, 63-77.

Rundus, D., \& Atkinson, R. C. Rehearsal processes in free recall: A procedure for direct observation. Journal of Verbal Learning and Verbal Behavior, 1970, 9, 99-105.

Slamecka, N., \& Graf, P. The generation effect: Delineation of a phenomenon. Journal of Experimental Psychology: Human Learning and Memory, 1978, 4, 592-604.

Thorndike, E. L., \& LoRge, I. The teacher's word book of 30,000 words. New York: Teachers College Press, Columbia University, 1944.

Woodward, A. E., Bjork, R. A., \& Jongeward, R. H. Recall and recognition as a function of primary rehearsal. Journal of Verbal Learning and Verbal Behavior, 1973, 12, 608-617.

(Manuscript received for publication July 15, 1983.) 